\title{
Time-Frequency Analysis of Hot Rolling Using Manifold Learning ${ }^{\star}$
}

\author{
Francisco J. García ${ }^{1}$, Ignacio Díaz ${ }^{1}$, Ignacio Álvarez ${ }^{1}$, \\ Daniel Pérez ${ }^{1}$, Daniel G. Ordonez ${ }^{1}$, and Manuel Domínguez ${ }^{2}$ \\ 1 Universidad de Oviedo, Área de Ingeniería de Sistemas y Automática \\ \{fjgarcia, idiaz, ialvarez, dperez, dgonzalez\}@isa.uniovi.es \\ ${ }^{2}$ Universidad de León, Instituto de Automática y Fabricación \\ diemdg@unileon.es
}

\begin{abstract}
In this paper, we propose a method to compare and visualize spectrograms in a low dimensional space using manifold learning. This approach is divided in two steps: a data processing and dimensionality reduction stage and a feature extraction and a visualization stage. The procedure is applied on different types of data from a hot rolling process, with the aim to detect chatter. Results obtained suggest future developments and applications in hot rolling and other industrial processes.
\end{abstract}

Keywords: Hot rolling, dimensionality reduction, ISOMAP, spectrogram analysis.

\section{Introduction}

Ever since its inception in the end of sixteenth-century, metal rolling has become one of the most important industrial process in the world. Huge amounts of material and money are driven by the rolling industry, so it is so important to have a method not only for describing the observed behaviour of the process, but also for identifying the nature of a problem. Due to the high amount of variables involved, trying to find relationships among them appears to be a good aproach for dimensionality reduction (DR).

The selection of a set of variables that describes operating modes in industrial processes is closely related to DR. One of the most important DR technique is Principal Component Analysis (PCA) 1], described by Pearson in 1901. After PCA, other DR techniques have been proposed, such as Multidimensional Scaling (MDS) methods, Independent Component Analysis (ICA) 2] or SelfOrganizing Maps (SOM) 3 . However, in the last 10 years, a new trend in DR, based on nonlinear models, appeared and a new collection of algorithms have been proposed. These algorithms - called local embeddings- involve a local optimization by defining local models of the $k$ nearest neighbours and an alignement

\footnotetext{
* This work has been financed by a grant from Research Fund for Coal and Steel of the European Community and by the spanish Ministry of Science and Education and FEDER funds under grants DPI2009-13398-C02-01/02.
} 
in order to obtain the global coordinates of each model, usually implying a singular value decomposition (SVD). Some of the most known of these techniques are isometric feature mapping or ISOMAP 4, local linear embedding (LLE) [5], laplacian eigenmaps (LE) [6] and local tangent subspace alignment (LTSA) [7].

Faults appeared in rolling process provoke important economic losses to the final product, so it is necessary to detect them in an early state, which is usually performed by analizing the frequency content of signals. A common source of problems in rolling is chatter [8], which is an unexpected powerful vibration that affects the quality of rolled material by causing an unacceptable variation of thickness. The frequency band related to chatter appears at $100-300 \mathrm{~Hz}$, so monitoring this band is a possibility to detect it. A good way to visualize that information is the spectrogram, which provides a time-varying spectral representation that shows how the spectral density of the signal varies with time [9], making it possible to visually detect defects. Spectrograms can be understood as vectors of high dimensionality, making it possible to use them as inputs for DR techniques in order to divide them into different groups of data. In this paper we propose a method based on the use of embedding methods for the visulization of differences in spectrograms, applying it to vibration, speed and torque data of a hot rolling mill, showing not only the data structure, but also features of the different spectrograms. This paper is organized as follows: section 2 describes the method applied, section 3 shows a description of the experiment, section 4 submits the results and finally section 5 includes the conclusions obtained.

\section{Spectrogram Analysis Using Manifold Learning}

As proposed in [4] [5] [7, DR techniques are a good approach for image classification. From an industrial point of view, spectrograms can be understood as images of the process, because they provide time-frequency information about the state of the involved variables. The proposed method takes advantage of the analogy between spectrogram and image applying DR techniques. The procedure is divided into two parts: firstly spectrograms and ISOMAP projections of different coils are computed and secondly information about main features of process is visualized in a similar way to component planes [3] (See Fig. 1).

\subsection{Computation of ISOMAP Projections}

Due to the nature of the rolling process, it is common to do a spatial sampling of signals, where harmonics are independent from speed, therefore this procedure is performed at first. Considering a measured variable $y(t)$ at a regular time interval $T_{m}$, the sequence obtained is $y_{k}^{\prime}=y\left(t_{k}^{\prime}\right)$, where $t_{k}^{\prime}=k \cdot T_{m}$. Spatial resampling needs a speed measurement $\mathbf{v}=\left[v_{0}, v_{1}, \ldots, v_{k}\right]$, either linear or rotatory, to know the spatial points where the original data was sampled $\mathbf{x}=\left[x_{0}, x_{1}, \ldots\right]$. Afterwards, selecting a spatial sampling rate $\left(e_{m}\right)$ allows to obtain a signal regularly sampled at fixed space intervals - each $e_{m}-\mathbf{y}=\left[y_{0}, y_{1}, \ldots\right]^{T}$ where $y_{k}=y\left(t_{k}\right)$, with $t_{k}=0$ for $k=0$ and $t_{k}=t_{k-1}+\frac{e_{m}}{v_{k}}$ for $k=1,2, \ldots$. Since actual data 


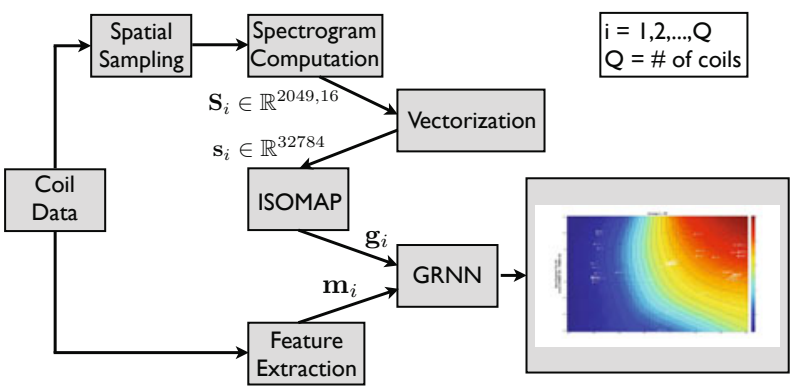

Fig. 1. Flowchart describing the method applied

is regular time sampled, the values of the spatial sampled data are obtained through linear interpolation.

The next step in our method is to compute the spectrogram of variables. Given a discrete signal $y_{k}=\left[y_{0}, y_{1}, \ldots, y_{n-1}\right]^{T}$, the Short-Time Fourier Transform (STFT) at a time $n$ is defined as $Y(\omega, m)=\sum_{n=-\infty}^{\infty} y_{n-m} \omega_{n} e^{-j \omega n}=$ $\sum_{n=0}^{R-1} y_{n-m} \omega_{n} e^{-j \omega n}$, where $\omega_{n}$ is a window function of length $R$. The spectrogram is a graphical display of the magnitude of $\operatorname{STFT},|Y(\omega, m)|$. This representation provides simplicity, robustness and ease of interpretation of the information contained in the frequency content. In Fig. 2, appearance of work roll (a), vibration (b), time (c) and spatial (d) spectrograms for a chattered coil are shown. Note that the strong $30 \mathrm{rev}^{-1}$ harmonic in the spatial spectrogram matches the periodicity of the marks in the work rolls for a whole turn, regardless the rolling speed. This makes such kind of representation a proper invariant descriptor of spatial phenomena typically involved in chatter effect.

Because of this type of visualization, it is possible to stablish a logical analogy between spectrogram and image. After spectrogram computation, the obtained matrix is converted into a vector with the aim to apply a DR technique. Being $\mathbf{S}$

(a)

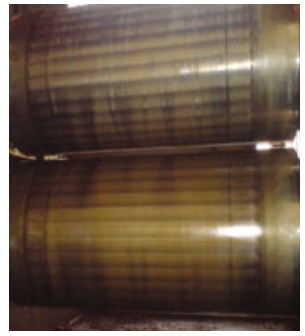

(b)

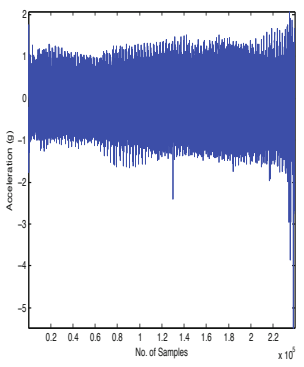

(c)

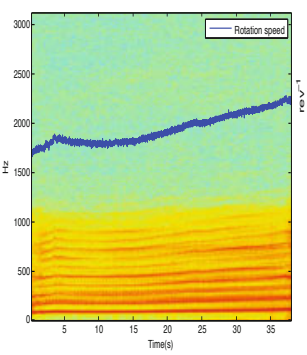

(d)

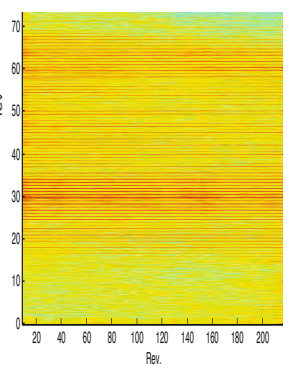

Fig. 2. Typical case of chattered coil 
the spectrogram matrix of dimension $\left(\frac{R}{2}+1\right) \times M$, each spectrogram can be represented as a vector $\mathbf{s}=\left[s_{0}, \ldots, s_{p}\right]^{T}$, where $s_{r+(m-1)\left(\frac{R}{2}+1\right)}=S_{r, m}$. The ISOMAP algorithm [4] was applied to $\mathbf{s}$. The ISOMAP algorithm is a DR technique where geodesic distance on a weighted graph is incorporated with the classical scaling, obtaining low-dimensional embedding of a set of high-dimensional data points.

\subsection{Feature Extraction and Visualization}

The first step of this stage is the extraction of relevant features from the signals being analysed. Considering a variable $y_{k}$ of length $N$, a windowed Fast Fourier Transform (FFT) -as an efficient algorithm for Discrete Fourier Transform (DFT), avoiding Gibbs effect- is applied in order to obtain its spectral information, as $Y_{i}=\sum_{k=0}^{N-1} w(k) y_{k} e^{-j 2 \pi k / N}$, for $i=0, \ldots, N-1$, where $Y_{i}$ is a complex sequence describing the amplitudes and phases of the signal harmonics and $w(k)$ is a Hanning window. In this case, a $N$-length window is used, so there is only one value of energy for each harmonic. The energy in bands around $p$ specified center frequencies $f_{1}, f_{2}, \cdots, f_{p}$ with predefined bandwidths $B_{1}, B_{2}, \cdots, B_{p}$ can be computed by summing up the squares of the harmonics inside this bands, in order to obtain a $p$-dimensional feature vector $\mathbf{m}=\left[m_{1}, m_{2}, \ldots, m_{p}\right]^{T}$, where $m_{i}=\sqrt{\sum_{\frac{k}{N T} \in\left[f_{i}-\frac{B_{i}}{2}, f_{i}+\frac{B_{i}}{2}\right]}\left\|\mathbf{Y}_{k}\right\|^{2}}$. Finally, features extracted for each analysed signal can be arrange in a data matrix $\mathbf{M}=\left(\mathbf{m}_{n}\right)=\left[\mathbf{m}_{1}, \mathbf{m}_{2}, \ldots\right]$, where $\mathbf{m}_{n}$ represents the column feature vector of coil $n$. In addition to the frequency features, specific feature extraction derived from process domain knowledge can be added (see Section 3). The visualization of component planes requires the application of a interpolation algorithm to estimate the value of each feature at every point in the 2D visualization space. In this paper, the Specht's [10] algorithm, general regression neural network (GRNN) is applied to match the extracted features and ISOMAP projections.

\section{Experiment}

The method described here was applied to data from a hot rolling finishing facility. The analysed signals were acceleration at driver-side (Acc DS, $6250 \mathrm{~Hz}$ ), as a measure of vibration, the root mean square of velocity, both at operator side and at driver side (VRMS OS and VRMS DS, $10 \mathrm{~Hz}$ ), upper roll torque (Torque OS, $500 \mathrm{~Hz}$ ) and, finally, rotation speed of rolls (Rot Sp, $500 \mathrm{~Hz}$ ). Only data during rolling stage was analysed. Two states of rolling -non-chatter and chatter, which leads to low quality coils- were taken into account in order to understand the results obtained with this method. The total size of the sample is 111 coils, comprising both states of rolling. Due to the original difference in the sampling frequency between Acc DS and Rot Sp signals, Acc DS signal was decimated in order to have a unique sample rate. A spatial sample rate of $212.16 \mathrm{rev}^{-1}$ was selected using the minimum spatial distance between two samples in the data. Spectrogram computation was applied to Acc DS signal of every coil, with a window size of 4096 and an overlapping of $90 \%$. To have equal sized spatial spectrograms, only the first 30 revolutions of rolling were considered, resulting in a total 
size of 32784 elements for each spectrogram to be projected with the ISOMAP algorithm. The dimensionality reduction stage of spectrograms involved the application of the ISOMAP algorithm with $K=30$ and output dimensionality of 2. Apart from frequency features, the mean of the difference between each element of VRMS OS and VRMS DS was added, as $\mu_{v}=\frac{1}{N} \sum_{i=0}^{N-1}\left(v_{d_{i}}-v_{o_{i}}\right)$. Finally, component planes generation was computed with value of $\sigma=0.003$ in GRNN with the aim to obtain a smooth degradation of the color of the planes for the difference of chatter and non-chatter group.

\section{Results}

The application of the method obtains the ISOMAP projections shown in Fig. 3 (top). ISOMAP is able to make a clusterization of the high-dimensional input data, obtaining two clusters (chatter and non-chatter), being remarkable that coils number 14 and 15 which appear isolated. It can be observed that these two coils happen to appear just before a long series of chattered coils. Component planes of ISOMAP are visualized in Fig. 3 (bottom). In this representation, two different color zones dependent from the value of the feature analysed are distinguished. The effect of the $\sigma$ value stablished for the GRNN algorithm, smoothing the color in each plane, can also be observed. This representation provides information about the value of the feature analysed in each plane. In addition, component planes characterize two zones, related to chatter and non-chatter coils. Such representations combine the visualization of the cluster structure of the data with the visual display of the value of different features associated to
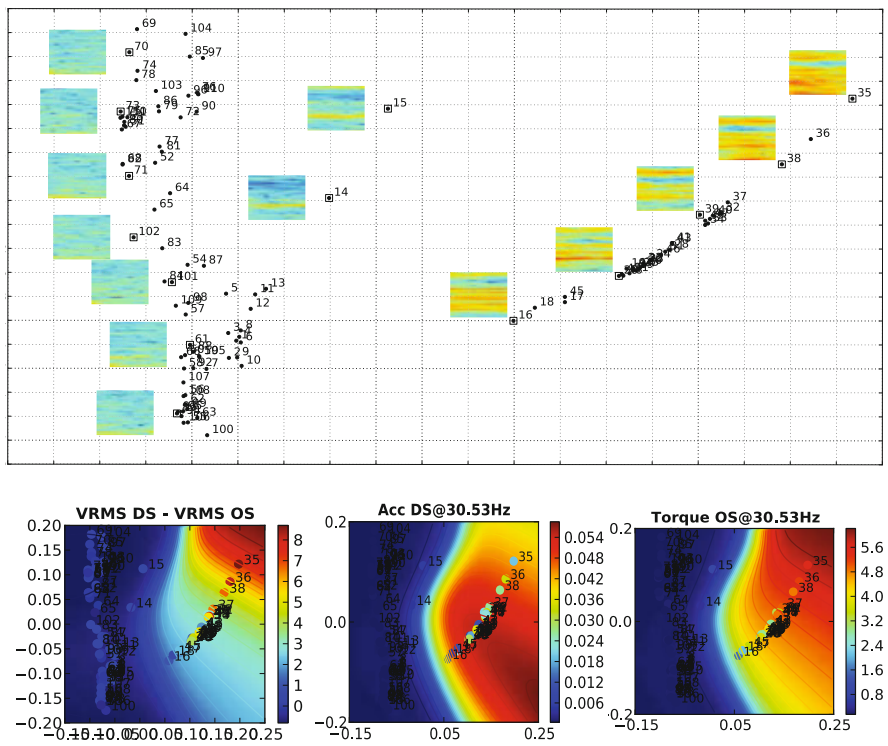

Fig. 3. ISOMAP projections and component planes of coils analysed 
each coil. Also, it is noteworthy the explanatory power in the definition of chatter of VRMS DS-VRMS OS, which is an unusual feature for detecting chatter.

\section{Conclusions}

In this paper we have proposed a method to explore the visualization of spectrograms in a low dimensionality as an use of ISOMAP, similar to image classification in 4. This method was applied to data from a hot rolling process in order to detect different states of rolling. The resulting projections show that different spectrograms produce low dimensional structures that can be efficiently unfolded with ISOMAP, distinguishing chatter, non-chatter and transition coils before chatter appears, which suggests that there is a difference in the frequency content of rolling that could be potentially used as to estimate when chatter could appear. Another contribution are component planes of ISOMAP, which provides information about analyzed features of the process, differentiating chatter and non chatter coils. These results suggest the potential use of the proposed method not only in hot rolling, but also in other kinds of batch process monitoring or in fault detection where time-frequency behavior of one or more variables bear information on process quality or fault conditions.

\section{References}

1. Jollife, I.: Principal component analysis (1986)

2. Hyvarinen, A.: Survey on independent component analysis. Neural Computing Surveys 2, 94-128 (1999)

3. Kohonen, T.: Self-Organizing Maps, 3rd extended edn. Springer Series in Information Sciences, vol. 30. Springer, Heidelberg (2001)

4. Tenenbaum, J.B., de Silva, V., Langford, J.C.: A global geometric framework for nonlinear dimensionality reduction. Science 290, 2319-2323 (2000)

5. Roweis, S.T., Saul, L.K.: Nonlinear dimensionality reduction by locally linear embedding. Science 290, 2323-2326 (2000)

6. Belkin, M., Niyogi, P.: Laplacian eigenmaps for dimensionality reduction and data representation. Neural computation 15(6), 1373-1396 (2003)

7. Zhang, Z., Zha, H.: Principal manifolds and nonlinear dimension reduction via local tangent space alignment. SIAM Journal of Scientific Computing 26(1), 313338 (2004)

8. Drevermann, J., Fieweger, M., Jckel, I.: Prozess-und zustandsberwachung an einem fertiggerst. In: Akida 2010, Aachen, Germany (November 17-18, 2010)

9. Boashash, B. (ed.): Time-Frequency Signal Analysis and Processing A Comprehensive Reference. Elsevier Science, Amsterdam (2003)

10. Specht, D.F.: A general regression neural network. IEEE Transactions on Neural Networks 2(6), 568-576 (1991) 